\title{
Detection of deafness-causing mutations in the Greek mitochondrial genome
}

\author{
Haris Kokotas $^{\mathrm{a}, *}$, Maria Grigoriadou ${ }^{\mathrm{a}}$, George S. Korres ${ }^{\mathrm{a}}$, Elisabeth Ferekidou ${ }^{\mathrm{a}}$, Dimitrios Kandiloros ${ }^{\mathrm{b}}$, \\ Stavros Korres ${ }^{\mathrm{c}}$ and Michael B. Petersen ${ }^{\mathrm{a}}$ \\ a Department of Genetics, Institute of Child Health, 'Aghia Sophia' Children's Hospital, Athens, Greece \\ ${ }^{\mathrm{b}}$ Department of Otorhinolaryngology - Head and Neck Surgery, Athens University Medical School, Attiko \\ Hospital, Athens, Greece \\ ${ }^{c}$ Department of Otorhinolaryngology - Head and Neck Surgery, Athens University Medical School, Hippokration \\ Hospital, Athens, Greece
}

\begin{abstract}
Mitochondrion harbors its own DNA, known as mtDNA, encoding certain essential components of the mitochondrial respiratory chain and protein synthesis apparatus. mtDNA mutations have an impact on cellular ATP production and many of them are undoubtedly a factor that contributes to sensorineural deafness, including both syndromic and non-syndromic forms. Hot spot regions for deafness mutations are the MTRNR1 gene, encoding the 12S rRNA, the MTTS1 gene, encoding the tRNA for $\mathrm{Ser}^{(U C N)}$, and the MTTL1 gene, encoding the tRNA for Leu ${ }^{(U U R)}$. We investigated the impact of mtDNA mutations in the Greek hearing impaired population, by testing a cohort of 513 patients suffering from childhood onset prelingual or postlingual, bilateral, sensorineural, syndromic or non-syndromic hearing loss of any degree for six mitochondrial variants previously associated with deafness. Screening involved the MTRNR1 961delT/insC and A1555G mutations, the MTTL1 A3243G mutation, and the MTTS1 A7445G, 7472insC and T7510C mutations. Although two patients were tested positive for the A1555G mutation, we failed to identify any subject carrying the 961delT/insC, A3243G, A7445G, 7472insC, or T7510C mutations. Our findings strongly support our previously raised conclusion that mtDNA mutations are not a major risk factor for sensorineural deafness in the Greek population.
\end{abstract}

Keywords: 961delT/insC, A1555G, A3243G, A7445G, 7472insC, T7510C, Greece, mitochondrial DNA, mutation, sensorineural deafness

\section{Introduction}

Clinically significant hearing loss is present in at least 1.9 per 1,000 infants at birth and the prevalence rises to at least 2.7 per 1,000 by the age of four [1]. Genetic causes of hearing loss are estimated to account for $68 \%$ of cases expressed at birth and $55 \%$ of those expressed by the age of four. Genetic deafness is divided into syndromic forms, in which hearing loss is associated with a variety of other anomalies, and non-syndromic forms. The syndromic forms account for $30 \%$ of prelingual genetic deafness and include several hundred deafness syndromes, with the underlying genetic defect being

* Corresponding author: Haris Kokotas, Department of Genetics, Institute of Child Health, 'Aghia Sophia' Children's Hospital, Athens 11527, Greece. Tel.: +30 2132037 333; Fax: +30 2107700 111; E-mail: hkokotas@yahoo.gr. found in about 30 of them [2,3]. In non-syndromic genetic deafness of prelingual onset, autosomal recessive inheritance predominates $(80 \%)$, but autosomal dominant $(20 \%)$, X-linked $(1 \%)$, and mitochondrial $(<1 \%)$ forms have also been described [4]. Hearing loss can also be non-genetic in origin, induced by factors such as ototoxic drugs, perinatal infections, traumas etc. However, many cases are multifactorial, involving a collaboration of exogenous factors and mutations in single genes or several genes [5,6]. The severity of hearing loss may range from mild to profound and the damage in hearing may include all frequencies [6].

mtDNA is transmitted only through the matrilineal lineage [7]. Mitochondrial mutations are collected in the human mitochondrial genome database MITOMAP [8]. mtDNA mutations can be divided into large rearrangements and mutations limited to a few basepairs, the majority being point mutations. Most mtD- 
NA deletions, duplications, insertions, inversions, or other complex rearrangements involve several genes, as mitochondrial genes are located close to each other. Large deletions generally remove at least one tRNA gene and are therefore likely to cause a translational defect and dysfunction of multiple components of oxidative phosphorylation, and consequently the whole energy process [9]. Remarkably, specific mutations in tRNA genes can also lead to non-syndromic deafness, whereas other organ systems remain unaffected. This illustrates the limited genotype-phenotype correlation in mitochondrial disease. In the Caucasian population, at least $5 \%$ of postlingual, non-syndromic hearing impairment is due to known mtDNA mutations, thus representing the most frequent cause of hearing loss after the $35 \mathrm{delG}$ mutation in the GJB2 gene encoding connexin 26 [10]. In oriental populations the frequency might be even higher. Especially the mitochondrial genes MTRNRI and MTTS1 encoding the 12S rRNA and tRNA ${ }^{\operatorname{Ser}(U C N)}$ respectively, have been found to be associated with non-syndromic hearing loss. However, it is unclear which percentage of postlingual hearing loss is due to mtDNA mutations, as these mutations might be both constitutional but also acquired (somatic).

In this study of mtDNA deafness-causing mutations, we have selected specific point mutations of the mitochondrial genome reported to be associated with sensorineural hearing loss [6]. We have previously reported preliminary data for the A1555G [11] and A7445G [12] mtDNA mutations. Here we present our data of screening for the 961delT/insC, A1555G, A3243G, A7445G, 7472insC, and T7510C mtDNA mutations in the Greek deafness population, and we summarize our findings with this report and the previously reported data $[11,12]$.

\section{Materials and methods}

\subsection{Materials}

For the present study we recruited a large cohort of 656 unrelated individuals suffering from hearing impairment of childhood onset. These patients were referred to our Department for molecular analysis from all the major childhood deafness centers of Greece, from 1999 until 2010. We used an extensive questionnaire in order to exclude cases of non-Greek origin, unilateral or mixed hearing loss, otosclerosis, history of infections during pregnancy, bacterial meningitis, need of neona- tal ventilation, and history of head trauma associated with skull fracture and loss of consciousness. Cases with a long-term hospital intensive care treatment, poor DNA quality, and complete absence of clinical history were also excluded from the present study. By clinical genetic evaluation we separated the cases of syndromic deafness from the non-syndromic ones. Although the A1555G mtDNA mutation has been so far reported only in non-syndromic cases, in the present study we applied this protocol also in 35 cases of syndromic hearing impairment. Finally, our cohort consisted of 513 unrelated Greek patients with sensorineural, bilateral, syndromic and non-syndromic hearing loss of any degree. All cases were categorized in respect of their inheritance pattern, age of onset, and degree of hearing loss.

\subsection{Methods}

By pure tone audiometry the level of hearing loss was classified as mild (20-39 dB), moderate (40-69 $\mathrm{dB})$, severe (70-89 dB), or profound ( $>90 \mathrm{~dB})$, from an average at $500-2000 \mathrm{~Hz}$ in the better ear.

DNA was isolated from EDTA-anticoagulated blood samples by a standard salting out procedure [13]. All samples had been previously tested for the frequent GJB2 35delG mutation and the coding region of the GJB2 gene was sequenced in $35 \mathrm{delG}$ heterozygotes in order to investigate the possible existence of a second mutation in compound heterozygosity with the $35 \mathrm{delG}$ mutation (unpublished data).

Polymerase chain reaction (PCR) and restriction fragment length polymorphism (RFLP) protocols were recruited for the detection of the mtDNA deafness mutations. The methods used for the molecular analyses of this study are summarized in Table 1. For all mutations, the restriction fragments were visualized on $2 \%$ agarose gels under UV light. The PCR protocols used in this study are available upon request. Informed consent was obtained for all participating patients. The study was approved by the Ethics Committee of the Institute of Child Health, Athens, Greece (Reference approval No: ICH/G.15).

\section{Results}

\subsection{Clinical evaluation results}

A questionnaire evaluation of the patients revealed 24 cases of non-Greek origin, six cases of bacterial 
Table 1

Methods used for the molecular analyses of six mtDNA mutations associated with sensorineural hearing loss

\begin{tabular}{|c|c|c|c|c|c|}
\hline \multirow[t]{2}{*}{ Mutation } & \multirow{2}{*}{$\begin{array}{c}\text { Mitochondrial } \\
\text { DNA gene (GenBank, } \\
\text { GI:251831106) }\end{array}$} & \multirow[t]{2}{*}{ Primers } & \multirow[t]{2}{*}{ Enzyme* } & \multicolumn{2}{|c|}{ Amplified fragment (bp) } \\
\hline & & & & $\begin{array}{c}\text { Normal } \\
\text { sample (bp } \\
\end{array}$ & $\begin{array}{l}\text { Mutant } \\
\text { sample (bp) }\end{array}$ \\
\hline \multirow[t]{3}{*}{ 961delT/insC } & MTRNRI & F: 5'-AAGAGTGTTTTAGATCACCCGC-3' & BsrBI & \multirow{2}{*}{\multicolumn{2}{|c|}{2437}} \\
\hline & & R: 5'-CGGTAAGCATTAGGAATGCC-3' & & & \\
\hline & & & & $\begin{array}{l}1713,703, \\
21\end{array}$ & 1713,724 \\
\hline \multirow[t]{3}{*}{ A1555G } & MTRNRI & F: 5'-GCTCAGCCTATATACCGCCATCTTCAGCAA-3' & HaeIII & & 339 \\
\hline & & R: 5'-TTTCCAGTACACTTACCATGTTACGACTGG-3' & & & \\
\hline & & & & 216,123 & $216,93,30$ \\
\hline \multirow[t]{3}{*}{ A3243G } & MTTL1 & F: 5'-CCTCCCTGTACGAAAGGAC-3' & HaeIII & & 238 \\
\hline & & R: 5'-GCGATTAGAATGGGTACAATG-3' & & & \\
\hline & & & & $\begin{array}{l}169, \quad 37, \\
32\end{array}$ & $97,72,37,32$ \\
\hline \multirow[t]{3}{*}{ A7445G } & MTTS1 & F: 5'-GGATGCCCCCCACCCTACC-3' & XbaI & & 216 \\
\hline & & R: 5'-CCTACTTGCGCTGCATGTGCC-3' & & & \\
\hline & & & & 168,48 & 216 \\
\hline \multirow[t]{3}{*}{ 7472insC } & MTTS1 & $\begin{array}{l}\text { F: 5'-ACATAAAATCTAGACAAAAAAGGAAGGAAT- } \\
3 \text {, }\end{array}$ & $\mathrm{XcmI}$ & & 208 \\
\hline & & R: 5'-CTTCTATGATAGGGGAAGTAGCGT-3' & & & \\
\hline & & & & 208 & 168,40 \\
\hline \multirow[t]{3}{*}{$\mathrm{T} 7510 \mathrm{C}$} & MTTS1 & F: 5'-GGATGCCCCCCACCCTACC-3' & HinfI & & 216 \\
\hline & & R: 5'-CCTACTTGCGCTGCATGTGCC-3' & & & \\
\hline & & & & $\begin{array}{l}150,35, \\
31\end{array}$ & $98,52,35,31$ \\
\hline
\end{tabular}

* New England Biolabs, Inc, Ipswich, MA, USA.

Table 2

Distribution of the deafness patients according to the inheritance pattern, age of onset, and degree of hearing loss

\begin{tabular}{|c|c|c|c|c|}
\hline \multicolumn{2}{|l|}{ Cases } & Non-syndromic (\%) & Syndromic (\%) & Total $(\%)$ \\
\hline Inheritance & $\begin{array}{c}\text { Sporadic } \\
\text { Familial } \\
\text { Unknown }\end{array}$ & $\begin{array}{c}301(58.7) \\
138(26.9) \\
39(7.6)\end{array}$ & $\begin{array}{c}15(2.9) \\
2(0.4) \\
18(3.5)\end{array}$ & $\begin{array}{c}316(61.6) \\
140(27.3) \\
57(11.1)\end{array}$ \\
\hline Age of onset & $\begin{array}{l}\text { Prelingual } \\
\text { Postlingual } \\
\text { Unknown }\end{array}$ & $\begin{array}{c}378(73.7) \\
79(15.4) \\
21(4.1)\end{array}$ & $\begin{array}{c}15(2.9) \\
1(0.2) \\
19(3.7)\end{array}$ & $\begin{array}{c}393(76.6) \\
80(15.6) \\
40(7.8)\end{array}$ \\
\hline Degree of hearing loss & $\begin{array}{c}\text { Mild } \\
\text { Moderate } \\
\text { Severe } \\
\text { Severe/Profound } \\
\text { Profound } \\
\text { Unknown }\end{array}$ & $\begin{array}{c}27(5.3) \\
67(13.1) \\
115(22.4) \\
3(0.6) \\
209(40.7) \\
57(11.1)\end{array}$ & $\begin{array}{c}1(0.2) \\
3(0.6) \\
5(1.0) \\
-(-) \\
6(1.2) \\
20(3.9)\end{array}$ & $\begin{array}{c}28(5.5) \\
70(13.7) \\
120(23.4) \\
3(0.6) \\
215(41.9) \\
77(15.0)\end{array}$ \\
\hline
\end{tabular}

meningitis, four cases of rubella in pregnancy, two with toxoplasmosis in pregnancy, five cases with a long-term hospitalization, and four cases of cytomegalovirus infection during pregnancy. Forty nine cases had a complete lack of clinical history. Audiological examination revealed 33 cases of unilateral hearing loss, one case with mixed hearing loss, and two cases with otosclerosis. Laboratory testing showed that 13 cases failed to provide DNA of sufficient quality. Therefore, a total of 143, were excluded from this study. Among the 513 subjects included in the study, nineteen patients had been previously subjected to aminoglycosides. Nine- teen patients had been previously subjected to aminoglycosides.

Clinical genetic evaluation of patients with additional symptoms to hearing impairment indicated that, of the 513 subjects that were included in this study, 478 were non-syndromic and 35 were syndromic cases. The results from the categorization according to the inheritance pattern, age of onset, and degree of hearing loss are shown in Table 2.

\subsection{Molecular analyses results}

Previously reported screening for the A1555G mt- 
Table 3

Results from screening Greek patients for mtDNA deafness-causing mutations

\begin{tabular}{lccccc}
\hline Mutation & Available samples & Excluded & Tested & Result & Reference \\
\hline 961delT/insC & 656 & 147 & 509 & $0 / 509$ & This study \\
A1555G & 656 & 143 & 513 & $2 / 513(0.4 \%)$ & [11] and this study \\
A3243G & 656 & 150 & 506 & $0 / 506$ & This study \\
A7445G & 656 & 143 & 513 & $0 / 513$ & [12] \\
7472insC & 656 & 144 & 512 & $0 / 512$ & This study \\
T7510C & 656 & 149 & 507 & $0 / 507$ & This study \\
\hline
\end{tabular}

DNA mutation included 478 unrelated Greek patients with sensorineural, bilateral, non-syndromic hearing loss of any degree [11]. Two patients were found with the A1555G mtDNA mutation. Both were previously subjected to aminoglycosides, and one of them was heterozygous for the GJB2 35delG mutation [11]. Screening for the A7445G mtDNA mutation included 513 unrelated Greek individuals suffering either from prelingual or postlingual bilateral, sensorineural, syndromic or non-syndromic, hearing impairment. None of the patients was found to harbor the mutation [12]. For the detection of the 961delT/insC, A3243G, 7472insC, and T7510C mtDNA mutations, PCR amplification was not possible in four, seven, one and six samples respectively, due to poor DNA quality. None of the 35 cases of syndromic hearing impairment was found to harbor the A1555G mutation in the present study. All samples were tested negative for the 961delT/insC, A3243G, 7472insC, and T7510C mtDNA mutations. The results from the mutation screening are summarized in Table 3.

\section{Discussion}

mtDNA mutations occur spontaneously at a high rate and most changes are neutral polymorphisms without clinical significance [15]. Furthermore, there appears to be a class of slightly deleterious mutations that modify the risks of mitochondrial disease, as in a multifactorial model, and distinct mtDNA mutations may act synergistically to modulate disease expression [16]. Consequently, it might be difficult to determine the pathogenicity of a novel mtDNA variation. Mitochondrial disease is characterized by an impressive degree of variation, and both inter- and even intrafamilial variation is the rule rather than the exception [6]. It is proposed that the accumulation of mtDNA mutations and the subsequent cytoplasmic segregation of these mutations during life is an important contributor both to the ageing process and to several human degenerative diseases [17]. Environmental factors (diet, toxic factors, etc) might further contribute to the progressive break- down of mitochondrial function with age, resulting in a late-onset disease. Specific mtDNA mutations usually lead to progressive hearing loss with an age of onset varying from childhood to early adulthood.

To investigate the impact of known deafnesscausing mtDNA mutations in a Greek deafness population, we applied rapid PCR-RFLP protocols in a large cohort of syndromic and non-syndromic cases. Our research included mtDNA mutations that have been associated with non-syndromic (A1555G, A3243G, A7445G, 7472insC, T7510C) and syndromic (A3243G, A7445G, 7472insC) hearing impairment. We also included the 961delT/insC mtDNA variant which has been reported to be pathogenic in some studies and polymorphic in others.

The 961delT/insC mutation is a deletion of a single $\mathrm{T}$ with an insertion of a varying number of $\mathrm{Cs}$ in the MTRNRl gene. This mutation has been found in Chinese sporadic patients [18] and two Italian families with aminoglycoside ototoxicity $[19,20]$. In a recent study [21], a frequency of $2 \%$ among sensorineural hearing loss Japanese patients was reported, raising the possibility of a relatively high frequency of this mutation among hearing impaired patients. However, a similar frequency was found in control subjects and the hearing loss phenotype did not segregate with the mutation in families suggesting that the 961delT/insC mutation is non-pathogenic [21]. More recent studies have provided support that $961 \mathrm{delT} / \mathrm{insC}$ is a polymorphism, and might be common in Asian [22] and African populations [23].

The A1555G mutation affecting the MTRNR1 gene, which encodes the small subunit ribosomal RNA (12S rRNA), was the first mitochondrial mutation to be associated with non-syndromic hearing loss [24]. It is probably the most common mtDNA mutation causing hearing loss as it is present in 0.5 to $1 \%$ of hearingimpaired Caucasians [25,26], although a much higher prevalence has been reported among Spanish [27] and Asian [28] patients. The A1555G mutation has been found in many families with maternally inherited, nonsyndromic hearing loss, and also in sporadic patients 
with hearing loss after the use of aminoglycosides. The fact that none of our 35 syndromic cases was found to harbor the A1555G mtDNA mutation further supports the notion that the mutation is only associated with nonsyndromic hearing impairment. Our frequency of $0.4 \%$ (2/513) is similar to that reported in other European populations [25].

The A3243G mutation of the MTTL1 gene causes deafness and/or diabetes [29-31]. Neurosensory hearing loss is present in almost all patients. Many patients have retinopathy, myopathy, cardiomyopathy, encephalopathy, and kidney disease [31], providing a phenotypic continuum between mitochondrially inherited deafness and diabetes (MIDD) syndrome and myoclonic epilepsy, lactic acidosis, and stroke-like episodes (MELAS) syndrome. However, cases of nonsyndromic cases carrying the A3243G mtDNA mutation have been reported [32,33].

The A7445G mutation in the MTTS1 gene (called $\mathrm{T} 7445 \mathrm{C}$ in some earlier papers) has been reported in seven families to date [34-40]. The hearing loss in some of these families was non-syndromic, but in some families combined with palmoplantar keratoderma. The mutation was present in homoplasmic, heteroplasmic or combined homo- and heteroplasmic genotypes with large differences in the level of heteroplasmy even within families.

The 7472insC mutation was first reported in a Sicilian family [41] with sensorineural hearing impairment in some family members and a combination of deafness with ataxia, dysarthria and myoclonus in others. In a large Dutch family the 7472insC mutation was found to be responsible for non-syndromic hearing loss in all family members but one, who additionally had ataxia and myoclonus, suggesting that modifying secondary factors must account for the intrafamilial difference in penetrance of the neurologic abnormalities [42]. Additional four families with hearing loss combined with myoclonic epilepsy, ataxia, and cognitive impairment have been reported $[43,44]$. A recent study identified a family in which, all affected members presented with non-syndromic deafness [33].

The T7510C mtDNA mutation in the MTTS1 mitochondrial gene appears to be extremely rare, and has been so far reported in only three families from the United Kingdom [45], Spain [46], and North America [47] presenting non-syndromic hearing loss.

In this and in previous studies $[11,12]$ we have reported our results from screening a Greek deafness population for six mtDNA mutations associated with sensorineural hearing loss. Mitochondrial mutations lead- ing to syndromic and non-syndromic deafness have been previously reviewed [6]. Some of these mutations, which were not investigated in this study, including the A827G, T1095C, C1494T, and G7444A mtDNA mutations could potentially be involved in sensorineural deafness in the Greek population, and their impact remains to be elucidated in future studies.

Taking into consideration that mtDNA mutations accumulate with age and have been associated with postlingual hearing loss and presbyacusis [6], there is a chance that mtDNA mutation carriers will never get tested for mtDNA mutations. Our findings indicate that mtDNA deafness-causing mutations are not major risk factors, and that other genetic or environmental factors may be more important contributors to sensorineural deafness in this population.

\section{Acknowledgments}

The authors wish to thank the patients who participated in this research. We are also grateful to Dr. Xavier Estivill, Dr. Ignacio del Castillo, and Dr. Guy Van Camp for providing us with positive samples. This study was supported in part by a grant from Oticon Fonden, Denmark (MBP).

\section{References}

[1] C.C. Morton and W.E. Nance, Newborn hearing screening a silent revolution, New England Journal of Medicine $\mathbf{3 5 4}$ (2006), 2151-2164.

[2] C. Petit, Genes responsible for human hereditary deafness: symphony of a thousand, Nature Genetics 14 (1996), 385-391.

[3] B.L. Resendes, R.E. Williamson and C.C. Morton, At the speed of sound: gene discovery in the auditory system, American Journal of Human Genetics 69 (2001), 923-935.

[4] N.E. Morton, Genetic epidemiology of hearing impairment, Annals of the New York Academy of Sciences 630 (1991), 16-31.

[5] N. Fischel-Ghodsian, Mitochondrial deafness, Ear and Hearing 24 (2003), 303-313.

[6] H. Kokotas, M.B. Petersen and P.J. Willems, Mitochondrial deafness, Clinical Genetics 71 (2007), 379-391.

[7] R.E. Giles, H. Blanc, H.M. Cann et al., Maternal inheritance of human mitochondrial DNA, The Proceedings of the National Academy of Sciences U S A 77 (1980), 6715-6719.

[8] MITOMAP. A human mitochondrial genome database. http:// www.mitomap.org/MITOMAP. Last accessed August 22, 2010.

[9] F.N. Gellerich, M. Deschauer, Y. Chen et al., Mitochondrial respiratory rates and activities of respiratory chain complexes correlate linearly with heteroplasmy of deleted mtDNA without threshold and independently of deletion size, Biochimica et Biophysica Acta 1556 (2002), 41-52. 
[10] H.T. Jacobs, T.P. Hutchin, T. Käppi et al., Mitochondrial DNA mutations in patients with postlingual, nonsyndromic hearing impairment, European Journal of Human Genetics 13 (2005), 26-33.

[11] H. Kokotas, M. Grigoriadou, G.S. Korres et al., The A1555G mitochondrial DNA mutation in Greek patients with nonsyndromic, sensorineural hearing loss, Biochemical and Biophysical Research Communications 390 (2009), 755-757.

[12] H. Kokotas, M. Grigoriadou, G.S. Korres et al., Screening of a Greek deafness population for the A7445G mitochondrial DNA mutation, Molecular Genetics and Metabolism 100 (2010), 300-301.

[13] S.A. Miller, D.D. Dykes and H.F. Polesky, A simple salting out procedure for extracting DNA from human nucleated cells, Nucleic Acids Research 16 (1988), 1215.

[14] X. Estivill, N. Govea, E. Barceló et al., Familial progressive sensorineural deafness is mainly due to the mtDNA A1555G mutation and is enhanced by treatment of aminoglycosides, American Journal of Human Genetics 62 (1998), 27-35.

[15] S. Dimauro and G. Davidzon, Mitochondrial DNA and disease, Annals of Medicine 37 (2005), 222-232.

[16] M.X. Guan, N. Fischel-Ghodsian and G. Attardi, Nuclear background determines biochemical phenotype in the deafness-associated mitochondrial $12 \mathrm{~S}$ rRNA mutation, $\mathrm{Hu}$ man Molecular Genetics 10 (2001), 573-580.

[17] A.W. Linnane, S. Marzuki, T. Ozawa et al., Mitochondrial DNA mutations as an important contributor to ageing and degenerative diseases, Lancet 1 (1989), 642-645.

[18] C. Bacino, T.R. Prezant, X. Bu et al., Susceptibility mutations in the mitochondrial small ribosomal RNA gene in aminoglycoside induced deafness, Pharmacogenetics 5 (1995), 165172.

[19] N. Fischel-Ghodsian, Genetic factors in aminoglycoside toxicity, Annals of the New York Academy of Sciences 884 (1999), 99-109.

[20] R.A. Casano, D.F. Johnson, Y. Bykhovskaya et al., Inherited susceptibility to aminoglycoside ototoxicity: genetic heterogeneity and clinical implications, American Journal of Otolaryngology 20 (1999), 151-156.

[21] K. Kobayashi, T. Oguchi, K. Asamura et al., Genetic features, clinical phenotypes, and prevalence of sensorineural hearing loss associated with the 961delT mitochondrial mutation, $\mathrm{Au}$ ris Nasus Larynx 32 (2005), 119-124.

[22] J.W. Bae, K.Y. Lee, S.Y. Choi et al., Molecular analysis of mitochondrial gene mutations in Korean patients with nonsyndromic hearing loss, International Journal of Molecular Medicine 22 (2008), 175-180.

[23] S. Bardien, H. Human, T. Harris et al., A rapid method for detection of five known mutations associated with aminoglycoside-induced deafness, BMC Medical Genetics 13 (2009), 10:2.

[24] T.R. Prezant, J.V. Agapian, M.C. Bohlman et al., Mitochondrial ribosomal RNA mutation associated with both antibioticinduced and non-syndromic deafness, Nature Genetics 4 (1993), 289-294.

[25] S. Kupka, T. Tóth, M. Wróbel et al., Mutation A1555G in the $12 \mathrm{~S}$ rRNA gene and its epidemiological importance in German, Hungarian, and Polish patients, Human Mutation 19 (2002), 308-309

[26] R. Li, J.H. Greinwald, Jr., L. Yang et al., Molecular analysis of the mitochondrial $12 \mathrm{~S}$ rRNA and tRNASer(UCN) genes in paediatric subjects with non-syndromic hearing loss, Journal of Medical Genetics 41 (2004), 615-620.
[27] F.J. del Castillo, M. Rodríguez-Ballesteros, Y. Martín et al., Heteroplasmy for the $1555 \mathrm{~A}>\mathrm{G}$ mutation in the mitochondrial 12S rRNA gene in six Spanish families with non-syndromic hearing loss, Journal of Medical Genetics 40 (2003), 632-636.

[28] S. Usami, S. Abe, J. Akita et al., Prevalence of mitochondrial gene mutations among hearing impaired patients, Journal of Medical Genetics 37 (2000), 38-40.

[29] T. Kadowaki, H. Kadowaki, Y. Mori et al., A subtype of diabetes mellitus associated with a mutation of mitochondrial DNA, New England Journal of Medicine 330 (1994), 962968.

[30] B. Sepehrnia, T.R. Prezant, J.I. Rotter et al., Screening for mtDNA diabetes mutations in Pima Indians with NIDDM., American Journal of Medical Genetics 56 (1995), 198-202.

[31] P.J. Guillausseau, P. Massin, D. Dubois-LaForgue et al., Maternally inherited diabetes and deafness: a multicenter study, Annals of Internal Medicine 134 (2001), 721-728.

[32] M. Mancuso, M. Filosto, F. Forli et al., A non-syndromic hearing loss caused by very low levels of the mtDNA A3243G mutation, Acta Neurologica Scandinavica 110 (2004), 72-74.

[33] M. Lévêque, S. Marlin, L. Jonard et al., Whole mitochondrial genome screening in maternally inherited non-syndromic hearing impairment using a microarray resequencing mitochondrial DNA chip, European Journal of Human Genetics 15 (2007), 1145-1155.

[34] F.M. Reid, G.A. Vernham and H.T. Jacobs, A novel mitochondrial point mutation in a maternal pedigree with sensorineural deafness, Human Mutation 3 (1994), 243-247.

[35] N. Fischel-Ghodsian, T.R. Prezant, P. Fournier et al., Mitochondrial mutation associated with nonsyndromic deafness, American Journal of Otolaryngology 16 (1995), 403-408.

[36] K.B. Sevior, A. Hatamochi, I.A. Stewart et al., Mitochondrial A7445G mutation in two pedigrees with palmoplantar keratoderma and deafness, American Journal of Medical Genetics 75 (1998), 179-185.

[37] L. Martin, A. Toutain, C. Guillen et al., Inherited palmoplantar keratoderma and sensorineural deafness associated with A7445G point mutation in the mitochondrial genome, British Journal of Dermatology 143 (2000), 876-883.

[38] T.P. Hutchin, N.J. Lench, S. Arbuzova et al., Maternally inherited hearing impairment in a family with the mitochondrial DNA A7445G mutation, European Journal of Human Genetics 9 (2001), 56-58.

[39] H. Caria, T. Matos, R. Oliveira-Soares et al., A7445G mtDNA mutation present in a Portuguese family exhibiting hereditary deafness and palmoplantar keratoderma, Journal of the European Academy of Dermatology and Venereology 19 (2005), $455-458$.

[40] A. Maász, K. Komlósi, K. Hadzsiev et al., Phenotypic variants of the deafness-associated mitochondrial DNA A7445G mutation, Current Medicinal Chemistry 15 (2008), 1257-1262.

[41] V. Tiranti, P. Chariot, F. Carella et al., Maternally inherited hearing loss, ataxia and myoclonus associated with a novel point mutation in mitochondrial tRNASer(UCN) gene, Human Molecular Genetics 4 (1995), 1421-1427.

[42] K. Verhoeven, R.J. Ensink, V. Tiranti et al., Hearing impairment and neurological dysfunction associated with a mutation in the mitochondrial tRNASer(UCN) gene, European Journal of Human Genetics 7 (1999), 45-51.

[43] M. Jaksch, T. Klopstock, G. Kurlemann et al., Progressive myoclonus epilepsy and mitochondrial myopathy associated with mutations in the tRNA(Ser(UCN)) gene, Annals of Neurology 44 (1998), 635-640. 
[44] M. Schuelke, M. Bakker, G. Stoltenburg et al., Epilepsia partialis continua associated with a homoplasmic mitochondrial tRNA (Ser(UCN)) mutation, Annals of Neurology 44 (1998), 700-704.

[45] T.P. Hutchin, M.J. Parker, I.D. Young et al., A novel mutation in the mitochondrial tRNA(Ser(UCN)) gene in a family with non-syndromic sensorineural hearing impairment, Journal of Medical Genetics 37 (2000), 692-694.

[46] F.J. del Castillo, M. Villamar, M.A. Moreno-Pelayo et al.,
Maternally inherited non-syndromic hearing impairment in a Spanish family with the 7510T $>\mathrm{C}$ mutation in the mitochondrial tRNA(Ser(UCN)) gene, Journal of Medical Genetics 39 (2002), e82.

[47] V. Labay, G. Garrido, A.C. Madeo et al., Haplogroup analysis supports a pathogenic role for the $7510 \mathrm{~T}>\mathrm{C}$ mutation of mitochondrial tRNA ( $\operatorname{Ser}(\mathrm{UCN})$ ) in sensorineural hearing loss, Clinical Genetics 73 (2008), 50-54. 


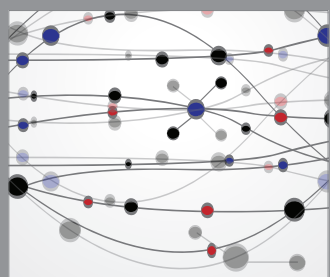

The Scientific World Journal
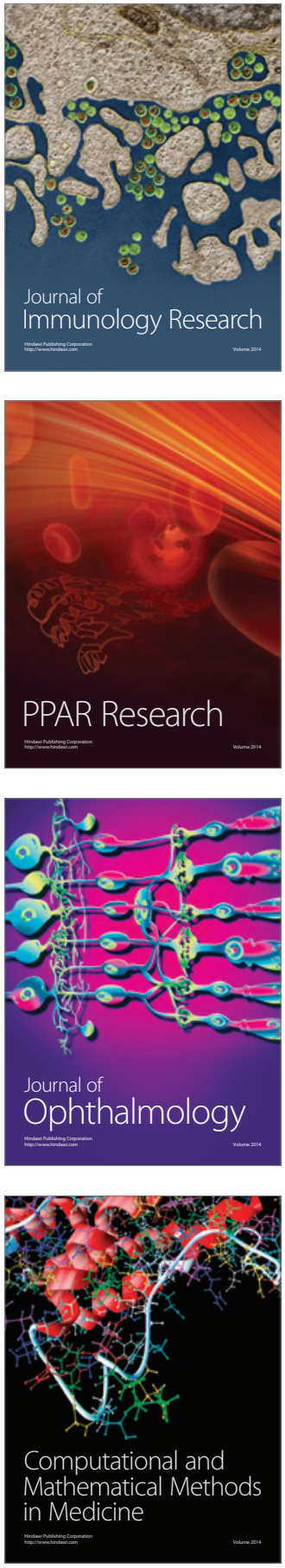

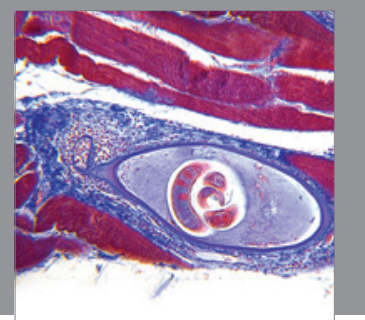

Gastroenterology

Research and Practice
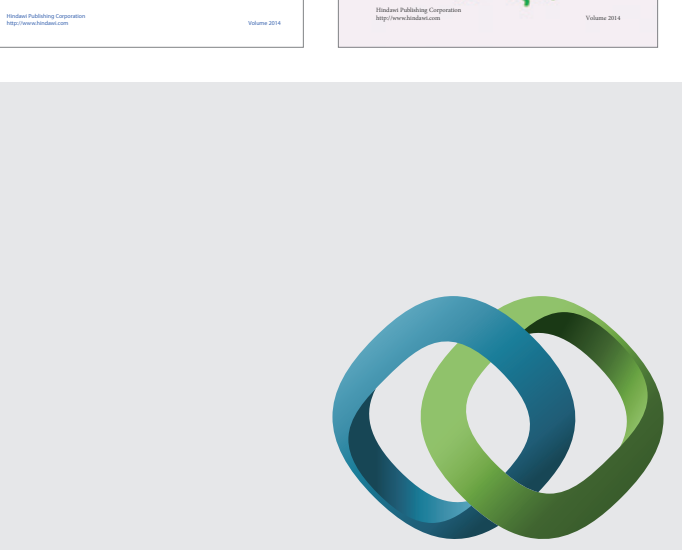

\section{Hindawi}

Submit your manuscripts at

http://www.hindawi.com
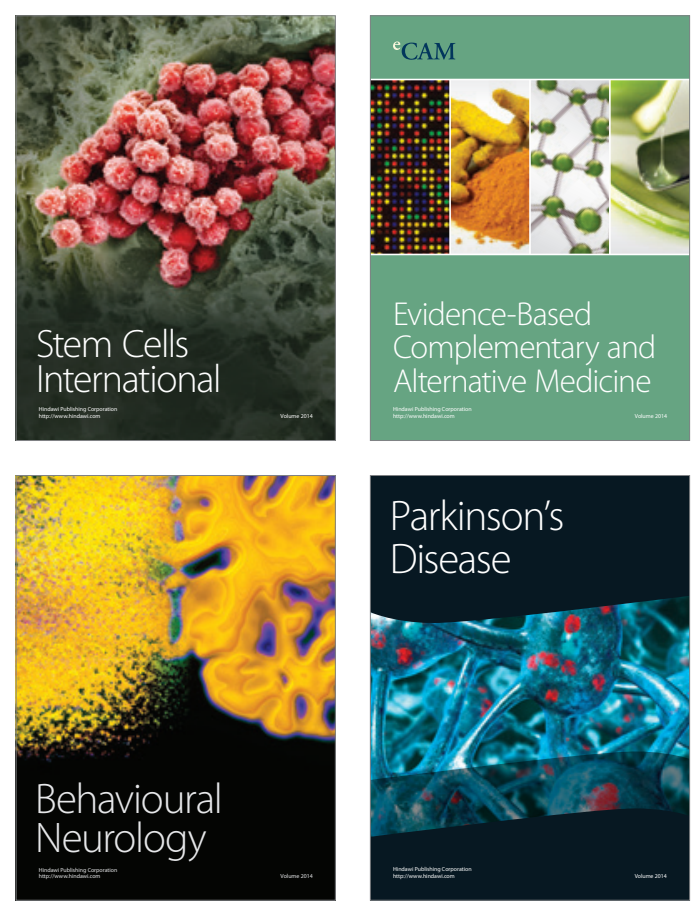

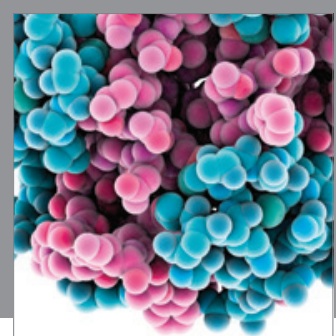

Journal of
Diabetes Research

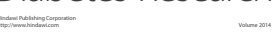

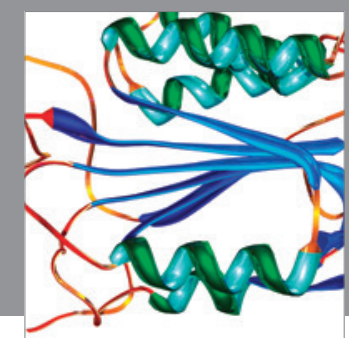

Disease Markers
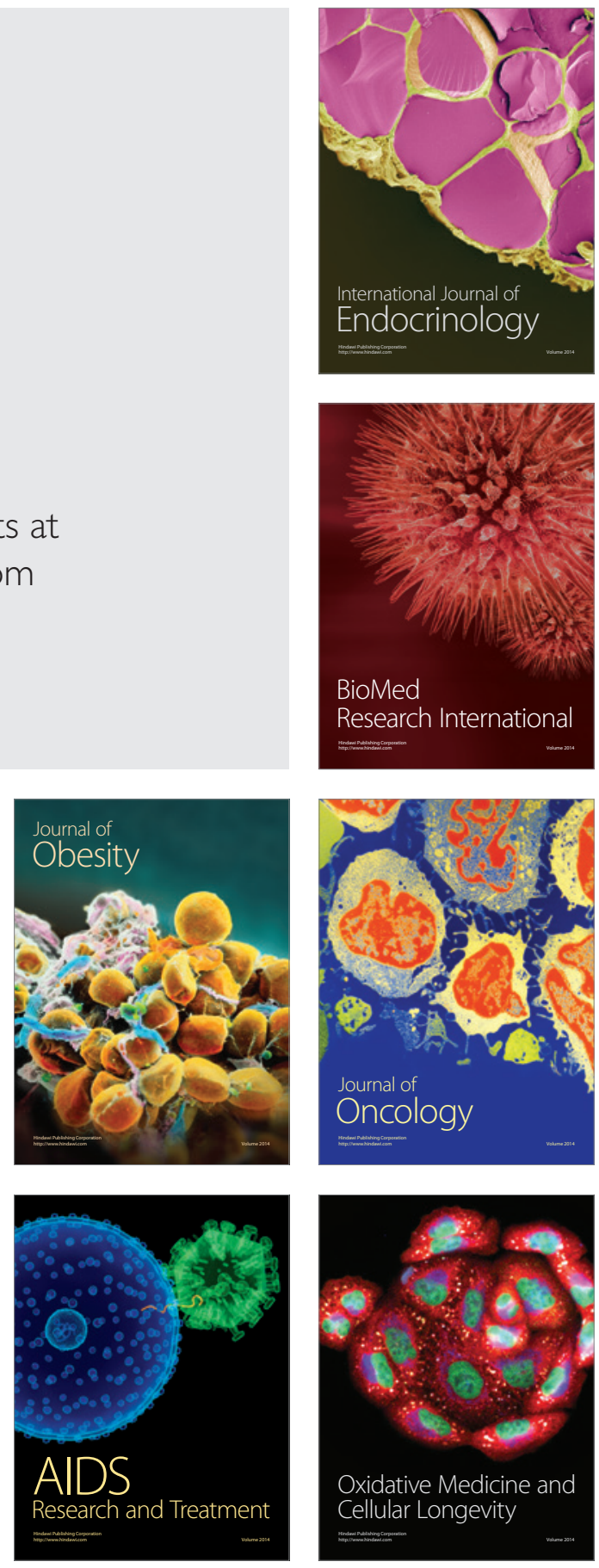